\title{
Clinical experience with apixaban in atrial fibrillation: implications of AVERROES
}

This article was published in the following Dove Press journal:

Research Reports in Clinical Cardiology

I 5 July 201 I

Number of times this article has been viewed

\author{
Raffaele De Caterina \\ Institute of Cardiology and Center \\ of Excellence on Aging, G d'Annunzio \\ University, Chieti, G Monasterio \\ Foundation, Pisa, Italy
}

\begin{abstract}
Atrial fibrillation is an extremely common arrhythmia, which substantially increases the risk of stroke and thromboembolism. Prevention of stroke and thromboembolism is therefore an important part of the management of atrial fibrillation. Guidelines until now have recommended that patients with atrial fibrillation receive some form of antithrombotic therapy, ie, a vitamin $\mathrm{K}$ antagonist or aspirin, with a preference for anticoagulants in most cases. However, current treatments are suboptimal, and despite the recommendations, many patients do not receive adequate thromboprophylaxis, because they are considered, for various reasons, "unsuitable" to receive a vitamin $\mathrm{K}$ antagonist. In this patient population, apixaban, a new oral anticoagulant inhibiting activated coagulation factor $\mathrm{X}$, administered in fixed doses and without anticoagulation monitoring, has undergone testing against aspirin in the recently published AVERROES trial. This paper addresses the strengths and limitations of this trial and the practical relevance of the new clinical information it provides.
\end{abstract}

Keywords: atrial fibrillation, apixaban, thromboprophylaxis

\section{Introduction}

Atrial fibrillation is the most common cardiac arrhythmia, ${ }^{1}$ and increases the risk of stroke and thromboembolism, on average, by five-fold. ${ }^{2}$ Strokes in atrial fibrillation are generally more severe and associated with greater mortality and disability than strokes from other causes. ${ }^{3}$ Therefore, prevention of stroke and thromboembolism is an important part of the management of atrial fibrillation. Guidelines until now have recommended that patients with atrial fibrillation receive some form of antithrombotic therapy, either as a vitamin $\mathrm{K}$ antagonist (VKA) or aspirin, with a preference for anticoagulants in most cases. ${ }^{4}$ However, these treatments are suboptimal, and despite recommendations, many patients do not receive adequate thromboprophylaxis. ${ }^{4}$ Apixaban is a new oral anticoagulant, which inhibits activated coagulation factor $\mathrm{X}$ (FXa). ${ }^{5}$ The first evidence of the efficacy of apixaban in atrial fibrillation has come from the recent AVERROES study. ${ }^{6}$ The practical relevance of these early clinical data is discussed here.

AVERROES was a randomized, double-blind, Phase III study assessing the efficacy and safety of apixaban $5 \mathrm{mg}$ bid versus aspirin $81-324 \mathrm{mg} /$ day for the prevention of stroke in 5600 patients with atrial fibrillation and at least one additional risk factor for stroke, who had failed or were considered unsuitable for VKA treatment. ${ }^{6}$ In April 2010, the data and safety monitoring board recommended early study termination because of clear benefit in favor of apixaban. The median duration of follow-up was 1.5 years. The primary outcome was stroke or systemic embolism. There were 51 primary outcome
Correspondence: Raffaele De Caterin Institute of Cardiology, Clo Ospedale SS Annunziata, Via dei Vestini,

66013 Chieti, Italy

Tel +39 087| 4I5I2

Fax +39 087I 4028I7

Email rdecater@unich.it 
events in those randomized to apixaban (1.6\% per year) and 113 in those randomized to aspirin (3.7\% per year) with a hazards ratio $(\mathrm{HR})$ of 0.45 (95\% confidence interval [CI]: $0.32-0.62 ; P<0.001)$. Mortality rates were $3.5 \%$ per year on apixaban and $4.4 \%$ per year on aspirin (HR: $0.79,95 \%$ CI: $0.62-1.02 ; P=0.07)$. There were $44(1.4 \%$ per year) major bleeds on apixaban and $39(1.2 \%$ per year $)$ on aspirin (HR: $1.13,95 \%$ CI: $0.74-1.75 ; P=0.57$ ). There were eleven intracranial bleeds on apixaban and 13 on aspirin. ${ }^{6}$ Thus, clear superiority of apixaban over aspirin was shown in terms of efficacy and with comparable safety. The clinical implications of the trial are briefly discussed.

\section{AVERROES in context}

One previous trial in a similar patient population, ie, ACTIVE A, ${ }^{7}$ tested the efficacy and safety of aspirin \pm clopidogrel in patients with atrial fibrillation. Aspirin + clopidogrel reduced the rate of major vascular events, in particular stroke, more than aspirin alone (relative risk [RR]: 0.72, 95\% CI: $0.62-0.84)$, but with an increased risk of major hemorrhage (RR: 1.56, 95\% CI: 1.28-1.89), and so had uncertain clinical benefit. ${ }^{7}$ Therefore, AVERROES has demonstrated that apixaban is superior to aspirin in terms of efficacy, with surprisingly similar safety. Based on the indirect comparison with ACTIVE A, one can conclude at the time of this writing that apixaban is the best alternative to aspirin in patients deemed unsuitable for a VKA.

\section{Strengths}

The strengths and important messages of AVERROES are:

- Its randomized, double-blind design

- The clear favorable outcome, with better efficacy and similar safety of apixaban versus aspirin

- Reassuring tolerability of the twice-daily dosing regimen for apixaban

- Confirmation that, in general, all new anticoagulants that completed or are completing Phase III trials (dabigatran etexilate, ${ }^{8}$ rivaroxaban [http://www.theheart.org/ article/1148785.do], and apixaban) all have strong activity in reduction of intracranial bleeds.

\section{Possible criticisms}

The main potential criticism of AVERROES concerns its inclusion criteria. Patients were eligible if they were 50 years of age or older and had atrial fibrillation documented in the 6 months before enrollment or by 12-lead electrocardiography on the day of screening. Patients also had to have at least one of the following risk factors for stroke: prior stroke or transient ischemic attack, age 75 years or older, arterial hypertension on treatment, diabetes mellitus on treatment, New York Heart Association functional class II heart failure or higher at the time of enrollment) a left ventricular ejection fraction $\leq 35 \%$, or documented peripheral arterial disease. In addition, patients could not be receiving VKA therapy because this had already been demonstrated to be unsuitable for them or because it was expected to be unsuitable. The reasons that VKA therapy was unsuitable for the patient had to be documented on the study case report forms.

Of the 5599 patients enrolled, reasons cited for unsuitability of VKA therapy included:

- International Normalized Ratio (INR) unable to be maintained in therapeutic range

- Adverse event(s) not related to bleeding during VKA therapy

- A serious bleeding event during VKA therapy

- Assessment that INR could not or was unlikely to be measured at requested intervals

- Expected difficulty in contacting patient for urgent change in dose of VKA

- Uncertainty about patient's ability to adhere to instructions regarding VKA therapy

- Concurrent medications that could alter activity of VKA

- Concurrent medications the metabolism of which could be affected by VKA

- Patient unable or unlikely to adhere to restrictions on alcohol and diet

- Hepatic disease

- Mild cognitive impairment, heart failure, cardiomyopathy or "other factors that could be associated with increased risk of VKA use"

- CHADS2 score $\leq 1$

- VKA therapy not recommended by the physician

- Other characteristics indicating risk of stroke too low to warrant treatment with VKA

- Patient refusal to take VKA

- Other reasons

All these reasons can be grouped into three broad categories:

- Assessment by the physician that thromboembolic risk (despite CHADS2 score $\geq 1$ being an inclusion criterion) was too low to warrant VKA therapy $(11 \%-12 \%)$

- Patient refusal to take VKA as the only reason for unsuitability (about 15\%)

- Physician-related assessment of unsuitability, about $75 \%$ of cases. 
One may question each of these choices. For example, current European Society of Cardiology guidelines underscore the importance of using VKA in low-risk categories $\left(\mathrm{CHADS}_{2} \text { VASC2 }=1\right)^{9}$ because of the demonstration that these patients also derive benefit from a VKA versus antiplatelet agents alone. ${ }^{10}$ Therefore, the category of "patients at too low risk to warrant VKA use" is currently shrinking more and more. Patient refusal to take a VKA is strongly influenced by the ability of the doctor to convey the message of the importance of a therapy to avert stroke. The vast majority of "unsuitability" was the physician's perception of an uncertain net clinical benefit (risk-benefit balance) for VKA therapy, which is to a large extent subjective and modifiable by other interventions. However, these proportions do reflect the current underuse of VKA in multiple registries. In the National Anticoagulation Benchmark Outcomes Report, retrospectively evaluating practices in hospitalized patients with atrial fibrillation ( $\mathrm{n}=945$ ), of $86 \%$ of patients eligible for warfarin, only $55 \%$ actually received it. ${ }^{11}$ This proportion was similar for both academic and community hospitals. In the Euro Heart Survey on atrial fibrillation, only $67 \%$ of patients eligible for VKA were actually prescribed this treatment, and $7 \%$ of eligible patients did not receive any form of antithrombotic treatment. ${ }^{12}$ Therefore, the population included in AVERROES represents a truly existing population in whom, for several reasons, VKA are not used, despite overwhelming evidence of their efficacy.

At this point, a few comments are opportune on how to position the population studied in AVERROES in the context of other ongoing trials of stroke prevention in patients with atrial fibrillation. Do the results of AVERROES render the aspirin + clopidogrel combination studied in ACTIVE-A obsolete in atrial fibrillation? Probably yes for most cases. The only exception to this is likely to be the population of patients with coexisting coronary artery disease receiving a stent, for whom dual antiplatelet therapy for some time ( 1 month in the case of a bare metal stent, 1 year with a drugeluting stent) is considered mandatory. Because stent thrombosis is considered mostly a platelet-dependent rather than a coagulation-dependent process, here the aspirin + clopidogrel combination, taking good care to prevent stent thrombosis and faring reasonably well in preventing stroke arising from atrial fibrillation, still has a place in therapy. In such cases, adding an anticoagulant (such as apixaban) to dual antiplatelet therapy has unproven benefits. In the broad category of patients with an acute coronary syndrome, as studied in the recently terminated APPRAISE-2 study, and while awaiting the results of the ongoing ATLAS-2 study with rivaroxaban, such a triple drug combination does not appear to be warranted. However, we still do not know whether patients with atrial fibrillation and a recently implanted stent may derive advantages from such a combination.

A more general question is whether the population of patients unsuitable for warfarin as tested in AVERROES will still exist in the era of new anticoagulants. Because most of the "unsuitability" depends on the multiple limitations of warfarin and VKA, and is not (in general) related to the whole category of novel anticoagulants, other agents, such as dabigatran etexilate, may find a place in such patients. However, this will need to follow the results of a properly designed trial. Finally, we have to realize that we will certainly not have head-to-head comparisons of novel agents for many years to come, and most of our indications and preferences for one agent over another will be based on indirect and cross-trial comparisons.

\section{Other anticoagulants in development for atrial fibrillation}

At the time of writing, dabigatran etexilate has been approved in the US and Canada for stroke prevention in atrial fibrillation, and approval is expected soon by the European Medicines Agency for European countries, based on the favorable results of RE-LY, a study testing dabigatran etexilate against warfarin in a broad spectrum of patients with nonvalvular atrial fibrillation. ${ }^{8}$ Results of a head-to-head comparison of the oral direct FXa inhibitor, rivaroxaban, against warfarin in patients with nonvalvular atrial fibrillation but at high risk of thromboembolism, showing noninferiority versus warfarin, have been presented at the 2010 American Heart Association scientific sessions (http://www.theheart.org/article/1148785. do). The trial met the noninferiority endpoint, with overall rates of bleeding for rivaroxaban being similar to those for warfarin, but with less intracranial hemorrhage. Another oral FXa inhibitor, edoxaban, is being tested in two doses against warfarin in atrial fibrillation, ie, ENGAGE AFTIMI 48, a randomized double-blind, double-dummy study in subjects with atrial fibrillation and a high risk of stroke (CHADS2 score $\geq 2$ ). The trial is estimated to be completed in March 2012. Thus, the current therapeutic options for stroke prevention in atrial fibrillation are expanding at a very rapid pace as of now, with exciting clinical research offering better tools than time-honored VKA therapy for stroke prevention in atrial fibrillation.

\section{Disclosure}

The author has been a Steering Committee Member of ACTIVE, AVERROES, and ARISTOTLE, and has repeatedly 
been a consultant and invited speaker at events organized by Sanofi-Aventis, Bristol-Myers Squibb, Pfizer, Boehringer Ingelheim, Bayer, Johnson \& Johnson, and Daiichi-Sankyo.

\section{References}

1. Heeringa J, van der Kuip DA, Hofman A, et al. Prevalence, incidence and lifetime risk of atrial fibrillation: the Rotterdam study. Eur Heart $J$. 2006;27:949-953.

2. Wolf PA, Abbott RD, Kannel WB. Atrial fibrillation as an independent risk factor for stroke: the Framingham Study. Stroke. 1991;22:983-988.

3. Jorgensen HS, Nakayama H, Reith J, Raaschou HO, Olsen TS. Acute stroke with atrial fibrillation. The Copenhagen Stroke Study. Stroke. 1996;27:1765-1769.

4. De Caterina R, Hylek EM. Stroke prevention in atrial fibrillation: current status and near-future directions. Am J Med. 2011. In press.

5. Carreiro J, Ansell J. Apixaban, an oral direct Factor Xa inhibitor: a waiting the verdict. Expert Opin Investig Drugs. 2008;17:1937-1945.

6. Connolly SJ, Eikelboom J, Joyner C, et al. Apixaban in patients with atrial fibrillation. $N$ Engl J Med. 2011;364:806-817.
7. Connolly SJ, Pogue J, Hart RG, et al. Effect of clopidogrel added to aspirin in patients with atrial fibrillation. $N$ Engl J Med. 2009;360: 2066-2078.

8. Connolly SJ, Ezekowitz MD, Yusuf S, et al. Dabigatran versus warfarin in patients with atrial fibrillation. $N$ Engl J Med. 2009;361: $1139-1151$.

9. Camm AJ, Kirchhof P, Lip GY, et al. Guidelines for the management of atrial fibrillation: the Task Force for the Management of Atrial Fibrillation of the European Society of Cardiology (ESC). Eur Heart J. 2010;31:2369-2429.

10. Healey JS, Hart RG, Pogue J, et al. Risks and benefits of oral anticoagulation compared with clopidogrel plus aspirin in patients with atrial fibrillation according to stroke risk: the atrial fibrillation clopidogrel trial with irbesartan for prevention of vascular events (ACTIVE-W). Stroke. 2008;39:1482-1486.

11. Waldo AL, Becker RC, Tapson VF, Colgan KJ. Hospitalized patients with atrial fibrillation and a high risk of stroke are not being provided with adequate anticoagulation. J Am Coll Cardiol. 2005;46:1729-1736.

12. Nieuwlaat R, Capucci A, Camm AJ, et al. Atrial fibrillation management: a prospective survey in ESC member countries: the Euro Heart Survey on Atrial Fibrillation. Eur Heart J. 2005;26:2422-2434.

\section{Publish your work in this journal}

Research Reports in Clinical Cardiology is an international, peerreviewed, open access journal publishing original research, reports, editorials, reviews and commentaries on all areas of cardiology in the clinic and laboratory. The manuscript management system is completely online and includes a very quick and fair peer-review system.

Submit your manuscript here: http://www.dovepress.com/research-reports-in-clinical-cardiology-journal
Visit http://www.dovepress.com/testimonials.php to read real quotes from published authors. 